\title{
Low density nuclear matter in effective field theory
}

\author{
S. Krewald ${ }^{1,3, a}$, P. Saviankou ${ }^{1}$, E. Epelbaum ${ }^{1,2}$, and Ulf-G. Meißner ${ }^{1,2,3}$ \\ 1 Institut für Kernphysik und Jülich Center for Hadron Physics, Forschungszentrum Jülich, D-52425 Jülich, Germany \\ 2 Universität Bonn, Helmholtz-Institut für Strahlen- und Kernphysik und Bethe Center for Theoretical Physics, Nußallee \\ 14-16, D-53115 Bonn, Germany \\ 3 Institute for Advanced Simulation, Forschungszentrum Jülich, D-52425 Jülich, Germany
}

\begin{abstract}
The two- and three-nucleon interaction derived in chiral effective field theory is used to obtain the binding energy of nuclear matter for small densities at next-to-leading (NLO) and next-to-next-to-leading orders $\left(\mathrm{N}^{2} \mathrm{LO}\right)$. The order $\mathrm{N}^{2} \mathrm{LO}$ is not yet sufficient to push the range of validity of the expansion beyond the empirical Fermi momentum of nuclear matter. A phenomenological extension of the interaction suggests that both effective three- and four-nucleon interactions are required for a description of the bulk properties of nuclear mater.
\end{abstract}

\section{Introduction}

Within the last two decades, effective field theory (EFT) has provided new methods for nuclear structure investigations. Both two- and three-nucleon interactions based on the most general chiral effective pion-nucleon Lagrangian have been developed utilizing Weinberg's power counting scheme. The phase shifts of the nucleon-nucleon interaction below the pion production threshold are reproduced with a precision that is comparable with the one achieved by the best phenomenological two-nucleon potentials, when pushing the expansion to next-to-next-tonext-to-leading order $\left(\mathrm{N}^{3} \mathrm{LO}\right)$, see Refs.[1,2]. These forces together with three-nucleon forces at $\mathrm{N}^{2} \mathrm{LO}$ have been applied to a cornucopia of reactions, as reviewed in Ref. [3]. Given these successes, the question arises whether effective field theory can contribute to the solution of the nuclear matter problem. First attempts in this direction have been undertaken by the Munich group, see e.g. Refs. [4,5] and references therein. We apply the NLO and $\mathrm{N}^{2} \mathrm{LO}$ versions of the chiral nucleon-nucleon interaction to nuclear matter. This differs from the Munich approach which concentrates on the development of a chiral effective interaction to be used exclusively in the nuclear medium with no direct link to the two-nucleon interaction in the vacuum. In Ref.[6], nuclear matter has been studied with a lowmomentum two-nucleon force $V_{\text {low-k }}$ derived from the Argonne $v_{18}$ potential via renormalization group techniques. It was found that the low-momentum interactions can be used in second order perturbation theory to describe nuclear matter, provided Pauli blocking effects due to the medium are properly included. This approach was recently extended to the $V_{\text {low-k }}$ versions of the chiral N3LO potentials [7]. Note, however, that chiral three-body forces at $\mathrm{N}^{2} \mathrm{LO}$ are included without appropriate running down

\footnotetext{
a e-mail: s.krewald@fz-juelich.de
}

to low momenta as the two-nucleon forces. Here, we follow a similar path but make use of effective field theory to maintain a systematic connection between the two- and many-nucleon forces at each order of the chiral expansion as reviewed in Ref. [3].

\section{The method}

The major ingredient of chiral effective field theory is power counting which allows to organize various contributions to the nucleon forces in a systematic way. In Ref.[8], chiral power counting was applied to derive nuclear forces from the most general effective chiral Lagrangian for pions and nucleons by eliminating the pionic Fock-space components via the FST-Okubo projection method $[9,10]$. The two-pion exchange diagrams relevant for the discussion of the properties of nuclear matter are shown in Fig. 1 up to NLO. The Weinberg-Tomozawa term generates a class of triangle diagrams (c) characterized by one internal nucleon line. Moreover, there are diagrams with two internal nucleon lines such as the crossed-box two pion exchange (a). Diagram (b) is an example of the so-called wave function renormalization graphs which are not two-nucleonreducible and therefore also generate contributions to the nuclear forces [11]. Finally, the so-called football diagram (d) does not involve nucleon propagators in the intermediate states.

In the nuclear medium, the two-nucleon interaction evaluated in the vacuum is modified by Pauli blocking effects. The most important and well-known Pauli blocking effect affects the intermediate two-nucleon states. For the chiral two-nucleon interaction, there are additional Pauli blocking effects due to the intermediate one- and two-nucleon propagators in the diagrams shown in Fig. 1.

Formally, its is trivial to take those blocking effects into account. The chiral forces are derived using the FST- 


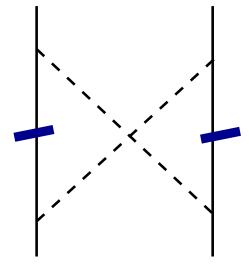

a)

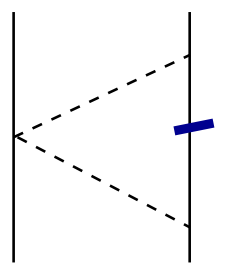

c)

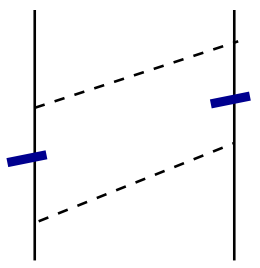

b)

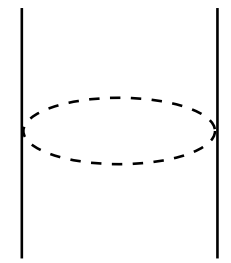

d)
Fig. 1. Two-pion exchange corrections to the chiral two-nucleon potential in NLO: a) crossed box, b) wave-function renormalization, c) triangle and d) football diagrams. Solid/dashed lines denote nucleons/pions. The thick lines indicate medium modifications due to Pauli blocking.

Okubo method of unitary transformations introducing a projector $\eta$ on the two-nucleon space and a projector $\lambda$ on the remaining part of the Fock space which consists of twonucleon and one- or multi-pion states. An effective Hamiltonian in the two-nucleon space is obtained by solving the decoupling condition

$$
\lambda(H-[A, H]-A H A) \eta=0,
$$

for the transition operator $A=\lambda A \eta$. In the nuclear medium, one has to introduce medium-modified projectors

$$
\begin{aligned}
& \bar{\eta}=\int d^{3} k_{1} \int d^{3} k_{2}\left(1-n\left(\mathbf{k}_{\mathbf{1}}\right)\right)\left(1-n\left(\mathbf{k}_{\mathbf{2}}\right)\right) \\
& \times\left|\mathbf{k}_{\mathbf{1}} \mathbf{k}_{\mathbf{2}}\right\rangle\left\langle\mathbf{k}_{\mathbf{1}} \mathbf{k}_{\mathbf{2}}\right|, \\
& \bar{\lambda}=\int d^{3} k_{1} \int d^{3} k_{2} \int d^{3} q_{\pi}\left(1-n\left(\mathbf{k}_{\mathbf{1}}\right)\right)\left(1-n\left(\mathbf{k}_{\mathbf{2}}\right)\right) \\
& \quad \times\left|\mathbf{k}_{\mathbf{1}} \mathbf{k}_{\mathbf{2}} \mathbf{q}_{\pi}\right\rangle\left\langle\mathbf{k}_{\mathbf{1}} \mathbf{k}_{\mathbf{2}} \mathbf{q}_{\pi}\right|,
\end{aligned}
$$

where $n(\mathbf{k})=1$ for $k \leq k_{F}$ and $n(\mathbf{k})=0$ for $k>k_{F}$ denotes the occupation probability of the level $k$. The momenta of the two nucleons and the pion are denoted by $\mathbf{k}_{\mathbf{1}}, \mathbf{k}_{\mathbf{2}}$ and $\mathbf{q}_{\pi}$, repectively.

The binding energy is obtained by summing all twonucleon reducible diagrams, as it is done in BruecknerHartree-Fock theory. Ref. [6] corresponds to the limit of the present approach for weak potentials. At NLO, genuine three-body forces are not yet included. The two-nucleon interactions derived in Ref. [2] are characterized by two regulators, the cut-off $\tilde{\Lambda}$ introduced in the spectral function representation of the two-pion exchange diagrams and the cut-off $\Lambda$ required for the non-perturbative renormalization of the Lippmann-Schwinger equation. The actual potentials are derived for a large set of regulators $\tilde{\Lambda}$ and $\Lambda$ in order to generate theoretical error bands for a given order of the expansion.

\section{Results at next-to-leading order}
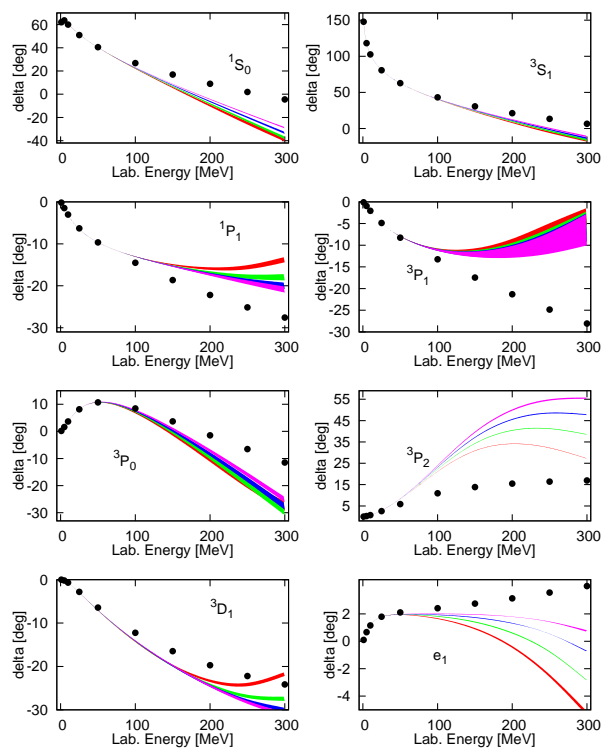

Fig. 2. Two-nucleon phase shifts versus the nucleon laboratory energy. The Lippman-Schwinger cut off $\Lambda$ takes the values 450 $\mathrm{MeV}$ (red), $500 \mathrm{MeV}$ (green), $550 \mathrm{MeV}$ (blue), and $600 \mathrm{MeV}$ (magenta). The filled circles correspond to the Nijmegen PWA results.

EFT represents the two-nucleon interaction at next-toleading order by one- and two-pion exchange diagrams, two S-wave contact terms accompanied by the low energy constants (LECs) $C_{S}$ and $C_{T}$, and seven contact terms for $\mathrm{P}$ - and D-waves, with the corresponding LECs $C_{1}, . . C_{7}$. There are no three-body interactions at this order. Details may be found in Ref. [12].

Fig. 2 shows the phases obtained for regulators with values $450 \mathrm{MeV} \leq \Lambda \leq 600 \mathrm{MeV}$ as compared to the Nijmegen phase shifts [13]. In the vicinity of the largest proton energy considered, $E_{\mathrm{Lab}}=300 \mathrm{MeV}$, the ${ }^{3} P_{2}$ phase shifts and the mixing parameter $e_{1}$ of the ${ }^{3} S_{1}-{ }^{3} D_{1}$ partial waves separate into clearly distinguishable bands characterized by the Lippmann-Schwinger cut-off $\Lambda$. With decreasing proton energy, the distances between those bands diminish until all bands merge near $50 \mathrm{MeV}$. In the case of the other P-waves and the S-waves, the bands merge at about $100 \mathrm{MeV}$. The experimental phase shifts are reproduced from threshold till approximately $50 \mathrm{MeV}$. For larger energies, the fit quality deteriorates. Although the description of the phase shifts at NLO leaves room for improvement in detail, the corresponding two-nucleon interaction shows interesting properties already at this order which makes an application to nuclear matter interesting. The ${ }^{1} S_{0}$ and ${ }^{3} S_{1}$ phase shifts become negative at $E_{\mathrm{Lab}}=$ $180 \mathrm{MeV}$ and $E_{\mathrm{Lab}}=250 \mathrm{MeV}$, respectively and show only 
a weak dependence on the cut-off momenta. In mesonexchange models, negative S-wave phase shifts at large energies are related to the repulsive core due to the $\omega$-meson which dominates the short distance physics and is assumed to play an essential role in producing the saturation of nuclear matter [14]. EFT treats long-range physics explicitly and summarizes the effects of short-range physics by contact interactions with their corresponding low-energy constants. Given the good reproduction of the experimental S-wave phase shifts, EFT may be expected to produce saturation of nuclear matter qualitatively already at NLO. A quantitative description of the empirical saturation point of nuclear matter may not be expected, however, because of the strong dependence of the higher partial waves on the regulators.

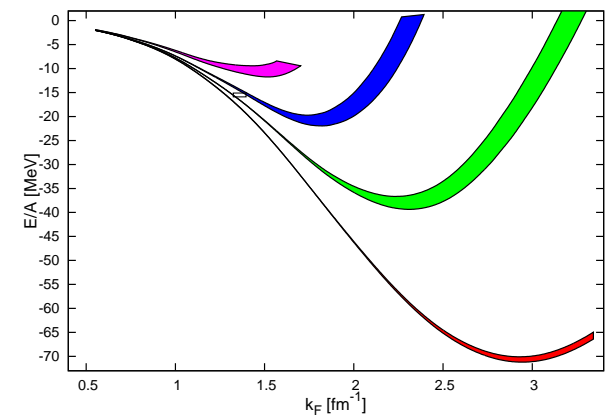

Fig. 3. Binding energy per particle of nuclear matter as a function of the Fermi momentum $k_{F}$ for the NLO potentials. The Lippman-Schwinger cut off $\Lambda$ takes the values $450 \mathrm{MeV}$ (red), $500 \mathrm{MeV}$ (green), $550 \mathrm{MeV}$ (blue), and $600 \mathrm{MeV}$ (magenta).

The binding energy per particle of nuclear matter is shown in Fig. 3 as a function of the Fermi momentum $k_{F}$. For small Fermi momenta, $k_{F} \leq 0.7 \mathrm{fm}^{-1}$, all saturation curves merge into a universal line, as required by the Hugenholtz-van Hove theorem [15]. A similar behaviour has been found in Ref. [6]. At larger values of the Fermi momenta, the saturation curves split into bands characterized by the Lippmann-Schwinger regulator $\Lambda$. For the cutoff $\Lambda=450 \mathrm{MeV}$, one obtains a saturation curve with closest distance to the empirical saturation point, but producing saturation at an unrealistically large Fermi momentum with extreme overbinding. The S-wave phase shifts produced with the cut-off $\Lambda=450 \mathrm{MeV}$ are more repulsive than the ones due to larger values of the cut-off, but on the other hand, $\Lambda=450 \mathrm{MeV}$ produces ${ }^{1} P_{1}$ and ${ }^{3} P_{1}$ phase shifts which are too attractive. Both $\mathrm{S}$ - and $\mathrm{P}$-wave phase shifts are reproduced better employing larger cut offs, such as $\Lambda=550 \mathrm{MeV}$ of $\Lambda=600 \mathrm{MeV}$. The corresponding saturation point moves down to $k_{F}=1.5 \mathrm{fm}^{-1}$.

\section{Results in next-to-next-to-leading order}

The phase shifts obtained at next-to-next-to-leading order are shown in Fig.4. The error bands corresponding to different values of the Lippman-Schwinger cut-off begin to

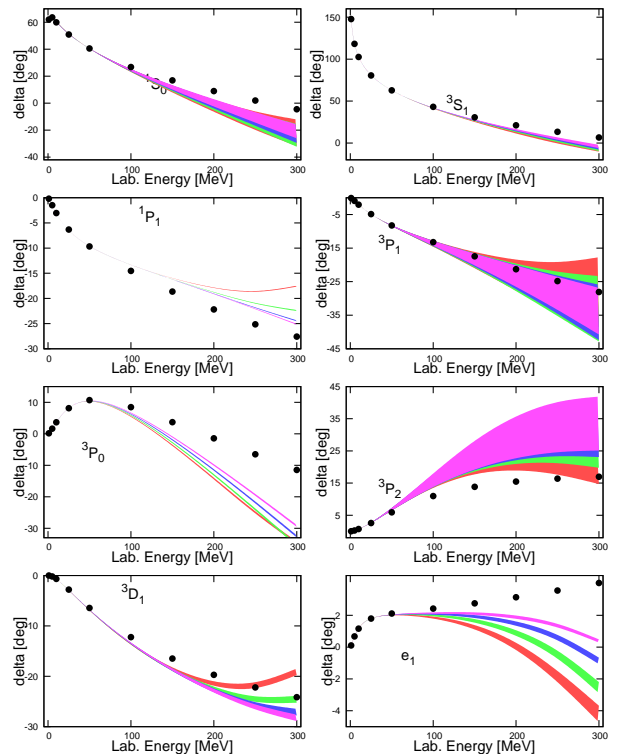

Fig. 4. Two-nucleon phase shifts versus the nucleon laboratory energy at $\mathrm{N}^{2} \mathrm{LO}$. The Lippman-Schwinger cut off $\Lambda$ takes the values $450 \mathrm{MeV}$ (red), $500 \mathrm{MeV}$ (green), $550 \mathrm{MeV}$ (blue), and 600 $\mathrm{MeV}$ (magenta).

merge even for the partial P- and D-waves. The fit of the ${ }^{1} S_{0}$ phase reduces the too repulsive behaviour of the ${ }^{1} S_{0}$ phase shift in NLO, which is a definite improvement. The ${ }^{3} P_{1}$ and ${ }^{3} P_{2}$ phase shifts are now much closer to the Nijmegen ones, showing more repulsion at higher energies than in the NLO approximation.

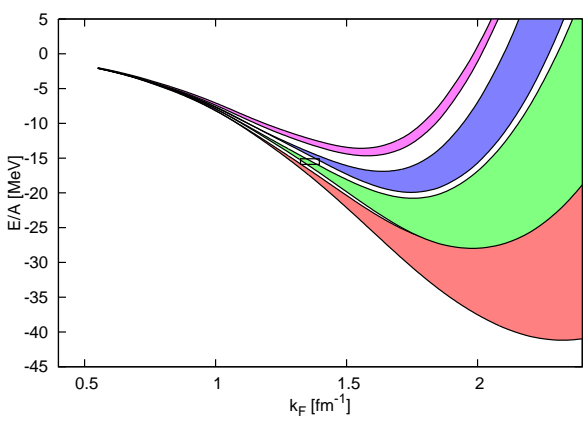

Fig. 5. Binding energy per particle of nuclear matter as a function of the Fermi momentum $k_{F}$ for the NNLO potentials. The Lippman-Schwinger cut off $\Lambda$ takes the values $450 \mathrm{MeV}$ (red), $500 \mathrm{MeV}$ (green), $550 \mathrm{MeV}$ (blue), and $600 \mathrm{MeV}$ (magenta).

The binding energy per particle of nuclear matter due to two-nucleon forces at order $\mathrm{N}^{2} \mathrm{LO}$ is shown in Fig. 5. The saturation curves are insensitive to the cut off values for Fermi momenta $k_{F} \leq 0.9 \mathrm{fm}^{-1}$ which improves the finding obtained at order NLO. For larger values of the Fermi momenta, the saturation curves split into bands characterized by the Lippmann-Schwinger regulator $\Lambda$, as at the order $\mathrm{N}^{2} \mathrm{LO}$, but now the range of binding energies covered by the various cut offs is more strongly restricted. At $k_{F}=1.5 \mathrm{fm}^{-1}$ which is well above the empirical sat- 
uration point, the cut off dependence introduces an uncertainty in the binding energy due to two-body interactions of about $10 \mathrm{MeV}$, which signals that the order $\mathrm{N}^{2} \mathrm{LO}$ does not yet suffice for an acceptable description of nuclear matter. Note that in the counting scheme of EFT, three-nucleon interactions appear for the first time at $\mathrm{N}^{2} \mathrm{LO}$. For each combination of regulators $\Lambda$ and $\tilde{\Lambda}$, the low energy constants $c_{D}$ and $c_{E}$ of the three-nucleon force have to be determined. In Fig. 6, the binding energy of nuclear matter obtained from the two-nucleon potential employing a Lippman-Schwinger cut-off $\Lambda=326 \mathrm{MeV}$ is shown by the dashed doubleddotted line. Clearly, this potential overbinds, as the empirical binding energy of $-16 \mathrm{MeV}$ is reached already at $k_{F}=1.2 \mathrm{fm}^{-1}$. For small cut offs, a perturbative treatment of the three-body interaction is justified [6]. We vary the strength of the force parameter $c_{D}$ in the range from -0.4 to 1.2 in order to study the effect of the three-body forces on the binding energy. For $c_{D}=1.2$, one obtains saturation of nuclear matter near the empirical Fermi momentum $k_{F}=1.36 \mathrm{fm}^{-1}$ but finds a binding energy of $-10 \mathrm{MeV}$, thus underbinding nuclear matter by $\sim 6 \mathrm{MeV}$.

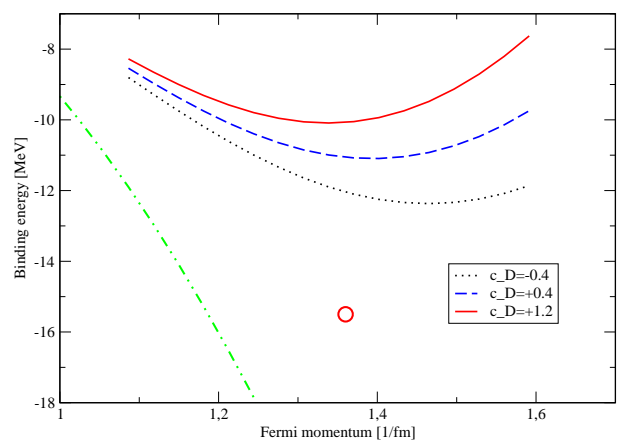

Fig. 6. Binding energy per particle of nuclear matter as a function of the Fermi momentum $k_{F}$ for the $\mathrm{N}^{2} \mathrm{LO}$ potential using a Lippman-Schwinger cut off $\Lambda=326 \mathrm{MeV}$. The dashed-double dotted green line refers to the $\mathrm{N}^{2} \mathrm{LO}$ result using only the twonucleon potential. The effects of the three-body forces is shown by the solid red line corresponding to $c_{D}=1.2 \mathrm{GeV}$. For comparison, we show additional calculations performed with smaller values of $c_{D}$. The empirical saturation point is indicated by the red circle.

After the inclusion of the three-body terms, the saturation point of nuclear matter is obtained near the empirical Fermi momentum $k_{F}=1.36 \mathrm{fm}^{-1}$, but nuclear matter is underbound by about $6 \mathrm{MeV}$, using a soft cut off. An increase of the binding energy goes together with an enhancement of the saturating Fermi momentum. Employing larger values of the Lippman-Schwinger cut off does not modify this conclusion. The binding energy of nuclear matter obtained from the $\mathrm{N}^{2} \mathrm{LO}$ potential corresponding to the regulators $\Lambda=550 \mathrm{MeV}$ and $\tilde{\Lambda}=500 \mathrm{MeV}$ is shown in Fig. 7. The parameters of the three-nucleon interaction are determined from a fit to the binding energies of light nuclei, $E\left({ }^{3} \mathrm{H}\right)=-8.482 \mathrm{MeV}, E\left({ }^{4} \mathrm{He}\right)=-27.88 \mathrm{MeV}$, and the alpha particle radius $r\left({ }^{4} \mathrm{He}\right)=1.959 \mathrm{fm}$, which leads to the values $c_{D}=-4.5$ and $c_{E}=-1.38$ [16]. Fol- lowing Ref. [6], we transform the three-body interation into a density-dependent two-body interaction. Below $k_{F}=$ $1 \mathrm{fm}^{-1}$, the contribution of the three-nucleon interaction is negligible. At larger Fermi momenta, the three-nucleon force produces more attraction than provided by the twonucleon interaction only. At larger densities, however, the

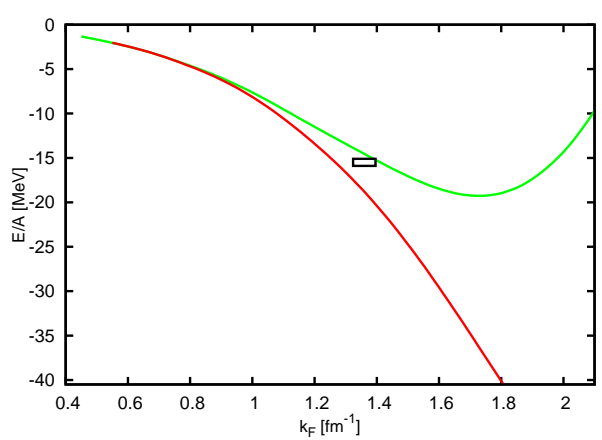

Fig. 7. Binding energy per particle of nuclear matter as a function of the Fermi momentum $k_{F}$ for the $\mathrm{N}^{2} \mathrm{LO}$ potential using a Lippman-Schwinger cut off $\Lambda=550 \mathrm{MeV}$. The red solid line refers to the $\mathrm{N}^{2} \mathrm{LO}$ result. For comparison, a calculation omitting the three-body forces is shown by the dashed green line. The empirical saturation point is indicated by the black box.

$\mathrm{N}^{2} \mathrm{LO}$ result becomes unrealistic, as the saturation point moves beyond $k_{F}=2 \mathrm{fm}^{-1}$. At a first look, it seems odd that the saturation point is not stable when the order of the expansion is increased. One has to realize, however, that there is a major difference between effective field theories and, for example, meson-exchange models of the nucleonic interaction. By their very nature, effective field theories are valid only at low momenta determined by scales of the degrees of freedom taken into account explicitly. In lowest order of the expansion, acceptable quantitative agreement with experiment is achieved only for very low momenta. With increasing order of the expansion, the range of quantitatively acceptable agreement with experiment increases. The effective field theory of the two-nucleon interaction provides a good example of this kind of convergence. Likewise, one may expect a quantitative rendering of the binding energy of nuclear matter only for small Fermi momenta in NLO. At order $\mathrm{N}^{2} \mathrm{LO}$, the contribution of the three nucleon interaction to the binding energy scales with an additional power of $k_{F}^{3}$, which becomes dominant at large $k_{F}$. Clearly, for large values of $k_{F}$, one has to include four-body interactions which make small contributions near the empirical saturation point, but guarantee the correct asymptotic behaviour of the binding energy at large densities. The results obtained in Figs. 6 and 7 show that effective field theory at order NLO and $\mathrm{N}^{2} \mathrm{LO}$ can be used to study nuclear matter for Fermi momenta below approximately $1 \mathrm{fm}^{-1}$. These orders of the expansion do not yet suffice to obtain cut-off independent binding energies of nuclear matter near the empirical saturation point. 
$19^{\text {th }}$ International IUPAP Conference on Few-Body Problems in Physics

\section{Effective density-dependent two-nucleon interaction}

The present investigation suggests that both three- and fournucleon interactions are required in an effective field theory of nuclear matter. Before proceeding to the order $\mathrm{N}^{3} \mathrm{LO}$, it may be worthwhile to consider the question whether density dependent modifications of the contact terms should be taken into account. To motivate such terms, consider a hypothetical effective field theory of the two-nucleon interaction which treats for example heavy mesons such as the $\omega$ and the $\rho$ explicitly. The low-energy constants defined in Ref. [12] should be obtained by integrating out the heavy mesons from the underlying hypothetical theory. When applied to nuclear matter, the short range part of the underlying theory changes, which leads naturally to a density dependent modification of the low-energy constants. In order to estimate the coupling constants of such medium dependent corrections, we abandon the chiral counting scheme and construct a simplified two-nucleon interaction which consists of the leading order of the chiral interaction and only the contact terms of the next-to-leading interaction. Explicitly, the effective interaction reads:

$$
\begin{aligned}
V_{e f f}= & V_{O P E P}+V^{(0)}+V^{(2)}, \\
V_{O P E P}= & -\left(\frac{g_{A}}{2 F_{\pi}}\right)^{2} \tau_{\mathbf{1}} \cdot \tau_{\mathbf{2}} \sigma_{\mathbf{1}} \cdot \mathbf{q} \sigma_{\mathbf{2}} \cdot \mathbf{q}, \\
& \times\left(\frac{2}{q^{2}+M_{\pi^{ \pm}}^{2}}-\frac{1}{q^{2}+M_{\pi^{0}}^{2}}\right), \\
V^{(0)}= & C_{S}+C_{T} \sigma_{\mathbf{1}} \cdot \sigma_{\mathbf{2}} \\
V^{(2)}= & C_{1} \mathbf{q}^{2}+C_{2} \mathbf{k}^{2}+\left(C_{3} \mathbf{q}^{2}+C_{4} \mathbf{k}^{2}\right) \sigma_{\mathbf{1}} \cdot \sigma_{\mathbf{2}}, \\
& +i C_{5} \frac{1}{2}\left(\sigma_{\mathbf{1}}+\sigma_{\mathbf{2}}\right) \cdot \mathbf{k} \times \mathbf{q}, \\
& +C_{6} \sigma_{\mathbf{1}} \cdot \mathbf{q} \sigma_{\mathbf{2}} \cdot \mathbf{q}+C_{7} \sigma_{\mathbf{1}} \cdot \mathbf{k} \sigma_{\mathbf{2}} \cdot \mathbf{k},
\end{aligned}
$$

with $g_{A}$ the nucleon axial-vector couplng constant, $F_{\pi}$ the weak pion decay constant and $M_{\pi^{0}}\left(M_{\pi^{ \pm}}\right)$refer to the neutral (charged) pion mass. Further, $\mathbf{q}=\mathbf{p}^{\prime}-\mathbf{p}$ and $\mathbf{k}=$ $1 / 2\left(\mathbf{p}^{\prime}+\mathbf{p}\right)$ with $\mathbf{p}^{\prime}$ and $\mathbf{p}$ being the final and initial CMS momenta of the nucleons. The coefficients $C_{S}, C_{T}$, and $C_{i}, 1 \leq i \leq 7$ are determined by a fit to the phase shifts of the Nijmegen group. A regulator for internal pion loops is no longer required, but one has to employ a regulator for the solution of the Lippman-Schwinger equation. We used the same regulator as in the $\mathrm{N}^{2} \mathrm{LO}$ investigation, $\Lambda=$ $550 \mathrm{MeV}$. The results are shown in Table 1.

The corresponding phase shifts are shown in Fig. 8. The simplified interaction generates phase shifts similar to the ones obtained at next-to-leading order. The ${ }^{3} S_{1}$ phase shift is close to the Nijmegen values for all energies considered, the ${ }^{1} S_{0}$ phase shift reproduces the Nijmegen values for energies of the incident nucleon ranging from threshold up to $100 \mathrm{MeV}$, as was the case for the full NLO calculation, see Fig. 2. Likewise, both the NLO interaction and the simplified interaction overestimate the attraction in the ${ }^{3} P_{1}$ and ${ }^{3} P_{2}$ phase shifts.

Next, we simulate the effects of three- and four-body interactions by a density dependent modification of the ef-
Table 1. Coefficients of the contact terms

\begin{tabular}{|c|c|}
\hline & $C_{A}\left[10^{-4} \mathrm{GeV}^{-2}\right]$ \\
\hline$C_{S}$ & -0.009792 \\
$C_{T}$ & -0.000860 \\
\hline & $C_{i}\left[10^{-4} \mathrm{GeV}^{-4}\right]$ \\
\hline$C_{1}$ & +0.026292 \\
$C_{2}$ & +0.080494 \\
$C_{3}$ & -0.004566 \\
$C_{4}$ & -0.013290 \\
$C_{5}$ & +0.049980 \\
$C_{6}$ & -0.002209 \\
$C_{7}$ & -0.107220 \\
\hline
\end{tabular}
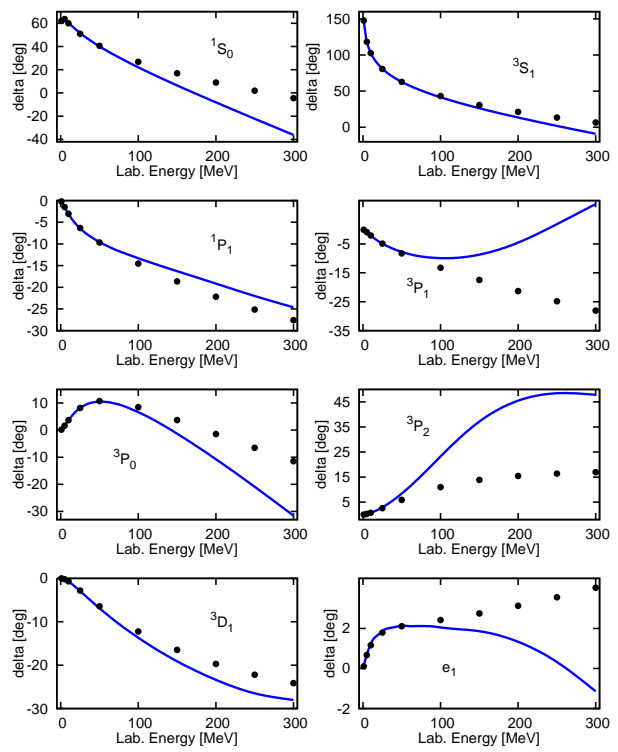

Fig. 8. Two-nucleon phase shifts versus the nucleon laboratory energy for a contact interaction, using a Lippman-Schwinger cut off $\Lambda=550 \mathrm{MeV}$

fective two-nucleon interaction $V^{(0)}$ as follows:

$$
\begin{aligned}
V^{(0)} & =C_{S}-\frac{1}{4} A\left[C_{S}-3 C_{T}\right] x(x-1) \\
& +\sigma_{1} \cdot \sigma_{2}\left(C_{T}+\frac{1}{4} A\left[C_{S}-3 C_{T}\right] x(x-1)\right) \\
x & =\left(\rho / \rho_{c}\right)^{\frac{1}{3}} .
\end{aligned}
$$

There are two parameters, $A$ and $\rho_{c}=2 k_{C}^{3} /\left(3 \pi^{2}\right)$. The empirical saturation point can be reproduced with the choice $A=0.025$ and $k_{C}=284 \mathrm{MeV}$. The ${ }^{1} S_{0}$ partial wave depends on the combination $C_{S}-3 C_{T}$ of the S-wave LECs and is changed by the modification above. The ${ }^{3} S_{1}$ partial wave is not affected, as it scales with $C_{S}+C_{T}$. Note that the low energy constant $C_{S}-3 C_{T}$ changes by less than $5 \%$ in the vicinity of $k_{F}=1.36 \mathrm{fm}^{-1}$. This finding gives hope that the convergence of the chiral expansion scheme may be rapid enough. Fig. 9 shows the binding energy per particle of nuclear matter as a function of the Fermi momentum $k_{F}$ for the simplified interaction. The two-body interaction which has been fitted to the phase shifts by itself leads to an unrealistically large saturation density. A 
similar finding has been obtained for the NLO interaction. The saturation found is not due to many-body forces, but exclusively due the repulsive components of the two-body interaction which are fixed by the phase shifts. In order to get a quantitative description of the saturation properties of nuclear matter, both three- and four-body forces have to be taken into account, however. At densities corresponding to the critical Fermi momentum $k_{C}$, the contributions of the three-body and four-body terms cancel. Below the critical density, the effective three-body interactions generate attraction in addition to the contributions from the two-body forces. At large densities, the four-body interactions generate repulsive contributions which stabilize the system. The compression modulus of nuclear matter is found to be $K=220 \mathrm{MeV}$. This is quite remarkabable, as the breathing mode of finite nuclei has been used by Blaizot to obtain empirical constraints on the compression modulus of nuclear matter[17], with a recent update by Colo et al. finding values ranging from $K=230 \mathrm{MeV}$ to $K=240 \mathrm{MeV}$ [18].

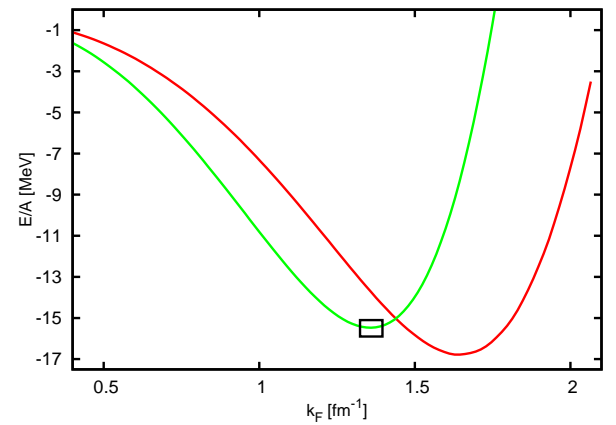

Fig. 9. Binding energy per particle of nuclear matter as a function of the Fermi momentum $k_{F}$ for a contact interaction, using a Lippman-Schwinger cut off $\Lambda=550 \mathrm{MeV}$ (red line). The solid (green) line shows the effect of a phenomenological density dependence. The empirical saturation point is indicated by the black box.

\section{Conclusions}

The two- and three-nucleon interactions derived from effective field theory at orders NLO and $\mathrm{N}^{2} \mathrm{LO}$ have been used to obtain the binding energies of nuclear matter in the limit of small densities. The binding energies of nuclear matter are insensitive to the cut-off parameters employed for Fermi momenta less than $k_{F}=0.9 \mathrm{fm}^{-1}$. The order $\mathrm{N}^{2} \mathrm{LO}$ does not yet allow to study the vicinity of the empirical saturation point of nuclear matter, $k_{F}=1.36 \mathrm{fm}^{-1}$. A simplified two-nucleon interaction supplemented by a phenomenological consideration of the effects of three- and four-nucleon interactions allows to simultaneously describe nucleon-nucleon phase shifts and the empirical binding energy and saturation density of nuclear matter.

\section{Acknowledgements}

We thank A. Nogga for providing the three-body constants. A helpful comment by Victor Tselyaev is gratefully acknowledged. We thank the DFG for partial support by the grant GZ:432RUS113/994/0-1. This work was further supported in parts by funds provided from the Helmholtz Association to the young investigator group "Few-Nucleon Systems in Chiral Effective Field Theory" (grant VH-NG222) and the Virtual Institute "Spin and strong QCD" (grant VH-VI-231). This work was further supported by the DFG (SFB/TR 16 "Subnuclear Structure of Matter") and the European Community-Research Infrastructure Integrating Activity "Study of Strongly Interacting Matter" (acr.: Hadron Physics2, Grant Agreement n. 227431) under the Seventh Framework Programme of the EU.

\section{References}

1. D. R. Entem and R. Machleidt, Phys. Lett. B $\mathbf{5 2 4}$ (2002) 93 [arXiv:nucl-th/0108057].

2. E. Epelbaum, W. Glöckle and U.-G. Meißner, Nucl. Phys. A 747 (2005) 362 [arXiv:nucl-th/0405048].

3. E. Epelbaum, H. W. Hammer and U.-G. Meißner, arXiv:0811.1338 [nucl-th].

4. N. Kaiser, S. Fritsch and W. Weise, Nucl. Phys. A 697 (2002) 255 [arXiv:nucl-th/0105057].

5. N. Kaiser, M. Muhlbauer and W. Weise, Eur. Phys. J. A 31 (2007) 53 [arXiv:nucl-th/0610060].

6. S. K. Bogner, A. Schwenk, R. J. Furnstahl and A. Nogga, Nucl. Phys. A 763 (2005) 59 [arXiv:nuclth/0504043].

7. S. K. Bogner, R. J. Furnstahl, A. Nogga and A. Schwenk, arXiv:0903.3366 [nucl-th].

8. E. Epelbaum, W. Glöckle and U.-G. Meißner, Nucl. Phys. A 637 (1998) 107 [arXiv:nucl-th/9801064].

9. N. Fukuda, K. Sawada and M. Taketani, Prog. Theor. Phys. 12 (1954) 156.

10. S. Okubo, Prog. Theor. Phys. 12 (1954) 603.

11. E. Epelbaum, W. Glöckle and U.-G. Meißner, Nucl. Phys. A 671 (2000) 295 [arXiv:nucl-th/9910064].

12. E. Epelbaum, Prog. Part. Nucl. Phys. 57 (2006) 654 [arXiv:nucl-th/0509032].

13. N. G. J. Stoks et al., Phys. Rev. C48 (1993) 792

14. H. A. Bethe, Annu.Rev.Nucl.Sci.21 (1971)93

15. N. M. Hugenholtz, Physica 23 (1957) 533

16. A. Nogga, private communication

17. J. P. Blaizot, Phys. Rep. 64 (1980)171.

18. G. Colo, N. VanGiai, J.Meyer, K. Bennaceur, P. Bonche, Phys. Rev. C70(2004)024307 